\title{
THE PILOCARPINE TEST
}

\author{
E. Muir.
}

This test, as described by Jurgensen and Milner, has been modified by A. Dubois and J. Degotte (1938).* They test for local sweating by the intradermal injection of a pilocarpine solution in small doubtful leprous patches, a second intradermal injection

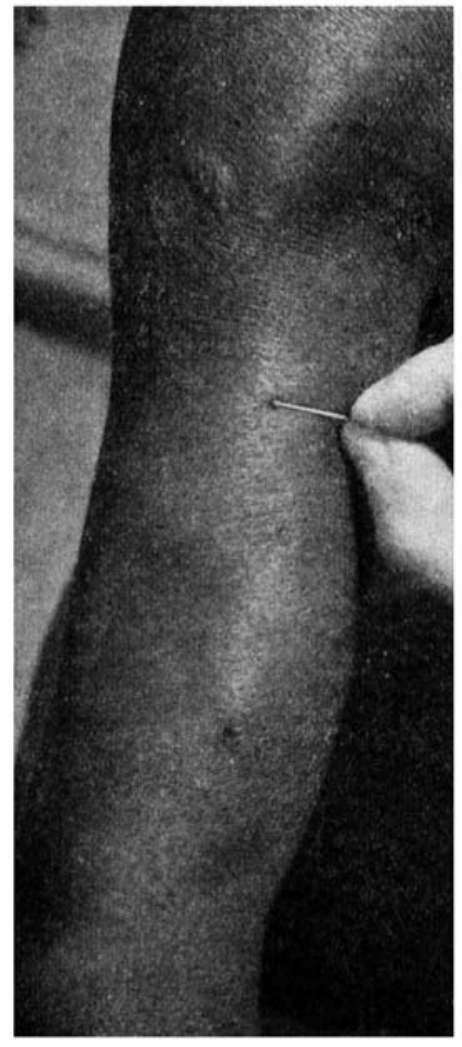

Fig. 3 .

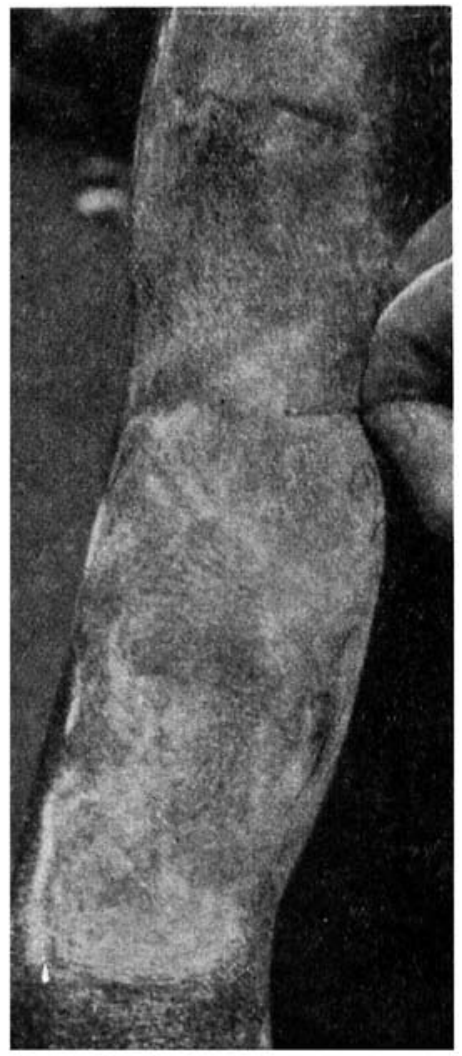

Fig. 4.

being given into normal skin as a control. Tincture of iodine is then painted over an area covering the site of both injections; and when this has dried powdered starch is dusted on. The positive inoculation remains dry, but there is sweating for a few millimetres round the control, thus bringing the iodine and starch in contact and forming a blue stain. The amount injected is 0.2 c.c. of a $I$ in $I, 000$ solution.

In the figs. 3 and 5 the wheals of the injections are seen in the

* Le test a la pilocarpine dans le diagnostic des macules lépreuses, par A. Dubois et J. Degotte. (Annales de la Société Belge de Médecine Tropicale. Tome XVIII, No. 3, Sept. 1938.) 
Fig. 5 .

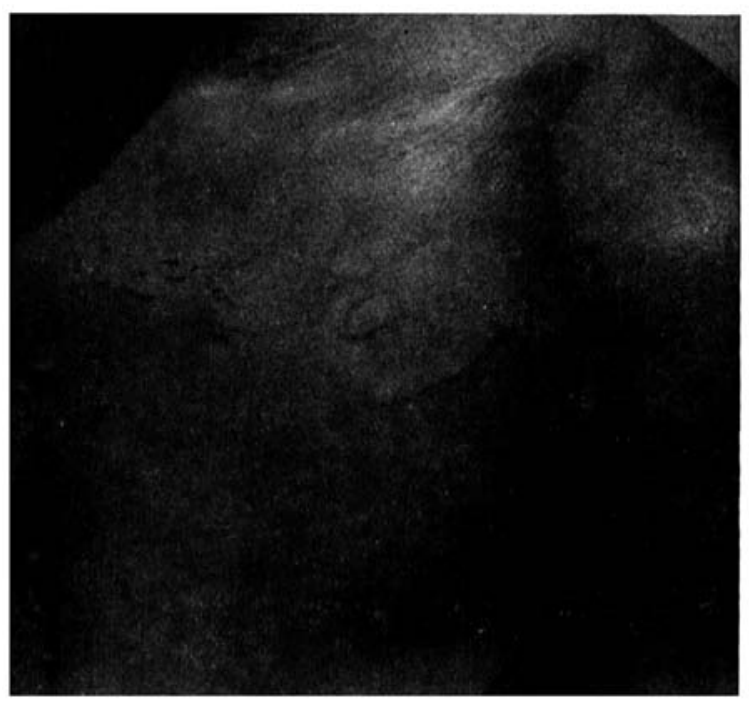

Fig. 6.

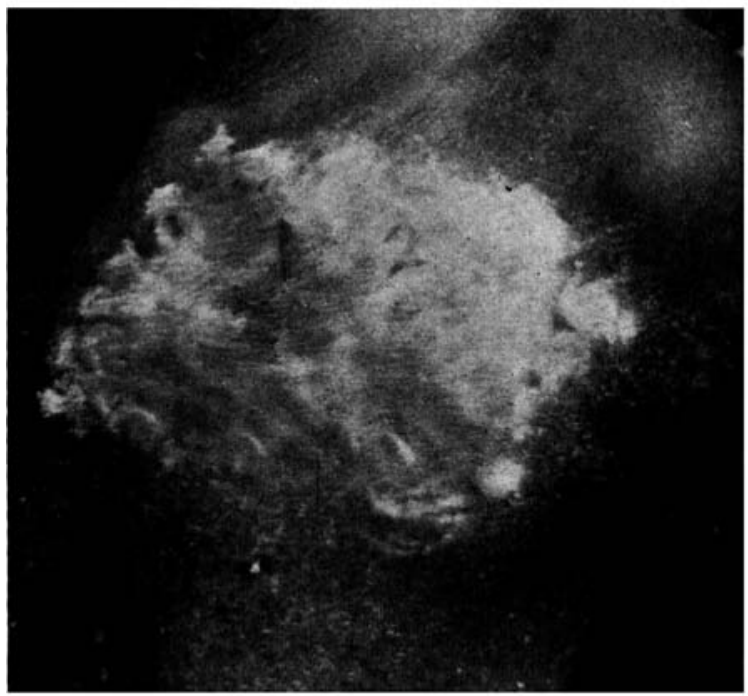

unpowdered skin. In figs. 4 and 6 showing powdered skin the controls are marked " I " and the wheals on suspected areas are marked " 2 ", and " 2 " and " 3 ".

While visiting the Belgian Croix Rouge station at Pawa, Congo Belge, I had an opportunity of studying this test in several of the slight cases of leprosy which are combed out by the very thorough survey which is carried out there. Prof. Dubois, while estimating progressive leprosy at 4 per cent., considers that the number would rise to 6 or 7 per cent. if the slight, frequently abortive, cases were included. The pilocarpine test is a valuable auxiliary means of diagnosis especially in early and slight cases. 